\title{
Weak Continuity and Compactness for Nonlinear Partial Differential Equations*
}

\author{
Gui-Qiang G. Chen \\ School of Mathematical Sciences, Fudan University, \\ Shanghai 200433, China. \\ Academy of Mathematics and Systems Science, \\ Chinese Academy of Sciences, Beijing 100190, China. \\ Mathematical Institute, University of Oxford, \\ Oxford, OX2 6GG, UK. \\ E-mail: Gui-Qiang.Chen@maths.ox.ac.uk
}

\begin{abstract}
This paper presents several examples of fundamental problems involving weak continuity and compactness for nonlinear partial differential equations, in which compensated compactness and related ideas have played a significant role. The compactness and convergence of vanishing viscosity solutions for nonlinear hyperbolic conservation laws are first analyzed, including the inviscid limit from the Navier-Stokes equations to the Euler equations for homentropic flow, the vanishing viscosity method to construct the global spherically symmetric solutions to the multidimensional compressible Euler equations, and the sonic-subsonic limit of solutions of the full Euler equations for multidimensional steady compressible fluids. The weak continuity and rigidity of the GaussCodazzi-Ricci system and corresponding isometric embeddings in differential geometry are revealed. Further references are also provided for some recent developments on the weak continuity and compactness for nonlinear partial differential equations.
\end{abstract}

\section{Introduction}

Nonlinear partial differential equations (PDEs) can be written as the following general form:

$$
\mathcal{N}[U]=0,
$$

${ }^{*}$ The research of Gui-Qiang Chen was supported in part by the UK EPSRC Science and Innovation Award to the Oxford Centre for Nonlinear PDE (EP/E035027/1), the UK EPSRC Award to the EPSRC Centre for Doctoral Training in PDEs (EP/L015811/1), the NSFC under a joint project Grant 10728101, and the Royal Society-Wolfson Research Merit Award (UK). 
where $\mathcal{N}[\cdot]$ is a nonlinear mapping, and $U$ is an unknown (scalar or vector) function that is called a solution if $U$ solves (1.1).

Two of the fundamental issues for nonlinear PDEs (1.1) are the following:

(i) Weak Continuity and Rigidity: Let $\left\{U^{\varepsilon}\right\}_{\varepsilon}>0$ be a sequence of exact solutions satisfying

$$
\left\{\begin{array}{l}
\mathcal{N}\left[U^{\varepsilon}\right]=0, \\
U^{\varepsilon} \rightarrow U \quad \text { in some topology as } \varepsilon \rightarrow 0 .
\end{array}\right.
$$

Issue 1: Does the limit function U satisfy

$$
\mathcal{N}[U]=0,
$$

or

$$
\tilde{\mathcal{N}}[U]=0
$$

for a different nonlinear mapping $\tilde{\mathcal{N}}[\cdot]$ associated with the original nonlinear mapping $\mathcal{N}[\cdot]$ and the solution sequence $\left\{U^{\varepsilon}\right\}_{\varepsilon>0}$ ?

Such an issue arises in rigidity problems in geometry, mechanics, among others.

(ii) Compactness and Convergence: Let $\left\{U^{\varepsilon}\right\}_{\varepsilon>0}$ be a sequence of approximate or multiscale solutions satisfying

$$
\left\{\begin{array}{l}
\mathcal{N}^{\varepsilon}\left[U^{\varepsilon}\right]=0, \\
U^{\varepsilon} \rightarrow U \text { in some topology as } \varepsilon \rightarrow 0 .
\end{array}\right.
$$

Issue 2: Does the limit function $U$ satisfy (1.3), or (1.4) for a different nonlinear mapping $\tilde{\mathcal{N}}[\cdot]$ associated with the nonlinear mappings $\mathcal{N}^{\varepsilon}[\cdot]$ and the solution sequence $\left\{U^{\varepsilon}\right\}_{\varepsilon>0}$ ?

This issue arises in the viscosity methods, relaxation methods, numerical methods, as well as problems for homogenization, hydrodynamic limits, search for effective equations, among others.

This paper presents several examples of these fundamental problems involving weak continuity and compactness for nonlinear PDEs, in which compensated compactness and related ideas, developed by Luc Tartar [83- 87] and François Murat [68- 72], have played a significant role; also see Tartar [89. In particular, in Section 2, we first analyze the compactness and convergence of vanishing viscosity solutions to hyperbolic conservation laws. In Section 3, we reveal the weak continuity and rigidity of the Gauss-Codazzi-Ricci system and corresponding isometric embeddings in differential geometry. Further references are also provided 
for some recent developments on the weak continuity and compactness for nonlinear PDEs. We finally remark that, as we will see in Sections $3-4$, many fundamental problems in this direction are still open, which require further new mathematical ideas, techniques, and approaches that deserve our special attention.

\section{Compactness and Convergence of Van- ishing Viscosity Solutions to Hyperbolic Conservation Laws}

Consider the following one-dimensional nonlinear hyperbolic conservation laws with form:

$$
\partial_{t} U+\partial_{x} F(U)=0, \quad U \in \mathbb{R}^{N},
$$

where $F: \mathbb{R}^{N} \rightarrow \mathbb{R}^{N}$ is a nonlinear mapping so that all the eigenvalues of $\nabla_{U} F(U)$ are real.

To solve these nonlinear PDEs, one of the important approaches is the viscosity method for which one honors the physical or designs an artificial $N \times N$ matrix function:

$$
D: \mathbb{R}^{N} \rightarrow M^{N \times N}, \quad D(U) \geq 0,
$$

so that

(i) $\partial_{t} U+\partial_{x} F(U)=\varepsilon \partial_{x}\left(D(U) \partial_{x} U\right)$ admits a global solution $U^{\varepsilon}(t, x)$ for each fixed $\varepsilon>0$;

(ii) $U^{\varepsilon}(t, x) \rightarrow U(t, x)$ in some topology as $\varepsilon \rightarrow 0$, and $U(t, x)$ is an entropy solution.

This method for the multidimensional case can be analogously formulated.

The idea of the vanishing viscosity method originates the philosophy of regarding the inviscid gas as the limit of viscous gases, which can date back in the 19th century, including the work by Stokes (1848), Rankine (1870), Hugoniot (1889), Rayleigh (1910), Taylor (1910), Weyl (1949), among others; also see Dafermos [27] and the references cited therein. This idea has played an essential role in developing the mathematical theory of hyperbolic conservation laws (such as discontinuous solutions, entropy conditions, existence, uniqueness, and solution behavior), as well as numerical methods and related applications (such as shock capturing, upwind, and kinetic schemes). This method becomes increasingly important, especially for understanding the recently observed non-uniqueness 
phenomena for the weak solutions satisfying the entropy equality for the multidimensional Euler equations ( $c f$. [28, 29]). On the other hand, the realization of this method is truly challenging in mathematics, since it involves several fundamental difficulties in analysis, including singular limits, nonlinearity, discontinuity, singularity, oscillation, cavitation, and concentration.

\subsection{Compactness and Convergence via $B V$-Estimates}

This compactness framework is based on the compactness theorem in $\mathrm{BV}$, which is a sufficient framework to ensure the strong compactness and convergence of exact/approximate solutions. On the other hand, achieving the BV-estimates of exact/approximate solutions is usually very challenging for the nonlinear systems, even though it is relatively easier for the scalar case.

\subsubsection{Scalar conservation laws}

Consider the Cauchy problem for scalar conservation laws $(N=1)$ :

$$
\partial_{t} U+\partial_{x} F(U)=\varepsilon \partial_{x x} U
$$

with the initial data $\left.U\right|_{t=0}=U_{0} \in B V \cap L^{\infty}(\mathbb{R})$. It can be shown that there exists $C$ independent of $\varepsilon$ such that the viscous solutions $U^{\varepsilon}=U^{\varepsilon}(t, x)$ of (2.3) satisfy

(i) Maximum principle: $\left\|U^{\varepsilon}\right\|_{L^{\infty}} \leq C$;

(ii) $B V$-estimate: $\left\|\partial_{x} U^{\varepsilon}\right\|_{L^{1}}+\left\|\partial_{t} U^{\varepsilon}\right\|_{L^{1}} \leq C$.

See Hopf [49, Oleinik [74, and Lax [55] for the one-dimensional case, and Vol'pert [90] and Kruzhkov [53] for the multidimensional case.

One of the approaches to achieve the BV-estimate is due to Vol'pert 90, which yields

$$
\begin{aligned}
& \partial_{t}\left(\left|\partial_{x} U^{\varepsilon}\right|\right)+\partial_{x}\left(F^{\prime}\left(U^{\varepsilon}\right)\left|\partial_{x} U^{\varepsilon}\right|\right) \leq \varepsilon \partial_{x x}\left(\left|\partial_{x} U^{\varepsilon}\right|\right), \\
& \partial_{t}\left(\left|\partial_{t} U^{\varepsilon}\right|\right)+\partial_{x}\left(F^{\prime}\left(U^{\varepsilon}\right)\left|\partial_{t} U^{\varepsilon}\right|\right) \leq \varepsilon \partial_{x x}\left(\left|\partial_{t} U^{\varepsilon}\right|\right)
\end{aligned}
$$

in the sense of distributions, leading to the BV-estimate.

Then the compactness theorem in BV implies the strong convergence of $U^{\varepsilon}(t, x)$.

Similar arguments can yield the $L^{1}$-equicontinuity of $U^{\varepsilon}$ directly, which is also a corollary of the $L^{1}$-stability and the comparison principle via Kruzhkov's method [53].

The same arguments also work for multidimensional scalar conservation laws (cf. [53, 90]); also see [11] for scalar conservation laws with memory. 


\subsubsection{Hyperbolic systems of conservation laws: $B V$-estimate via Glimm's approach}

Glimm 39] first developed a random choice method, the Glimm scheme, and derived the $B V$-estimate of the corresponding Glimm approximate solutions, based on the Glimm functional and corresponding wave interaction estimates. The techniques developed have been successfully employed to establish the global existence of solutions in $B V$ and analyze the behavior of solutions in $B V$ (structure, uniqueness, stability, and asymptotic behavior of solutions in $B V$ ) when the total variation of the initial data is small. Also see Glimm-Lax [40, DiPerna 31, Liu [59], Dafermos [27], and the references cited therein.

Theorem: For a strictly hyperbolic system (2.1) on $U$ in a neighborhood of a compact set $K \subset \mathbb{R}^{N}$, there exist constants $\delta>0$ and $C$ such that, if

$$
\text { Tot.Var. }\left\{U_{0}\right\}<\delta, \quad \lim _{x \rightarrow-\infty} U_{0}(x) \in K,
$$

then there exists a global solution $U(t, x)$ such that

$$
\text { Tot.Var. }\{U(t, \cdot)\} \leq C \text { Tot.Var. }\left\{U_{0}\right\} .
$$

Glimm's approach has been further employed to handle the fronttacking method and developed to analyze the $L^{1}$-stability of global solutions obtained by either the Glimm scheme or the front tracking method. See Bressan [5], Dafermos [27, Holden-Risebro [48, Liu-Yang 60], LeFloch [56], and the references cited therein. The approach has also been developed to analyze the well-posedness for two-dimensional steady supersonic Euler flows past a Lipschitz wedge in [24, 15].

\subsubsection{Hyperbolic system of conservation laws: BV-estimate for the artificial viscosity method}

Consider the following Cauchy problem for one-dimensional nonlinear hyperbolic systems of conservation laws with vanishing artificial viscosity (i.e. $\left.D(U)=I_{N \times N}\right)$ :

$$
\partial_{t} U+\partial_{x} F(U)=\varepsilon \partial_{x x} U
$$

and the initial data: $U(0, x)=U_{0}(x) \in B V\left(\mathbb{R}^{N}\right)$.

Theorem (Biachini-Bressan [4]): For a strictly hyperbolic system (2.1) on $U$ in a neighborhood of a compact set $K \subset \mathbb{R}^{N}$, there exist constants $\delta>0$ and $C_{j}, j=1,2,3$, such that, if $U_{0}$ satisfies (2.4), then, for any fixed $\varepsilon>0$, there exists a unique solution $U^{\varepsilon}(t, \cdot):=S_{t}^{\varepsilon} U_{0}(\cdot)$ of the Cauchy problem (2.5) such that 
(i) BV bound: Tot.Var. $\left\{S_{t}^{\varepsilon} U_{0}\right\} \leq C_{1}$ Tot.Var. $\left\{U_{0}\right\}$;

(ii) L $L^{1}$-stability: $\left\|S_{t}^{\varepsilon} U_{0}-S_{t}^{\varepsilon} V_{0}\right\|_{L^{1}} \leq C_{2}\left\|U_{0}-V_{0}\right\|_{L^{1}}$,

$$
\left\|S_{t}^{\varepsilon} U_{0}-S_{s}^{\varepsilon} U_{0}\right\|_{L^{1}} \leq C_{3}(|t-s|+|\sqrt{\varepsilon t}-\sqrt{\varepsilon s}|) .
$$

These imply the strong convergence and $L^{1}$-stability of the limit solution of (2.1).

The strategies to achieve the BV-estimate include the following steps:

(i) Employ the heat kernel to estimate the solution for $t \in\left[0, \tau_{\varepsilon}\right]$ :

$$
\left\|\partial_{x} U^{\varepsilon}(t, \cdot)\right\|_{L^{1}} \leq \kappa \delta,
$$

where $\kappa$ is small, independent of $\varepsilon$ and $\delta$.

(ii) Decompose $\partial_{x} U^{\varepsilon}$ along a suitable basis of unit vectors $\left\{\mathbf{r}_{1}, \cdots, \mathbf{r}_{N}\right\}$ :

$\partial_{x} U^{\varepsilon}=\sum v_{i}^{\varepsilon} \mathbf{r}_{i} \quad$ (sum of gradients of viscous travelling waves).

(iii) Derive a system of $N$ equations for these scalar components:

$$
\partial_{t} v_{i}^{\varepsilon}+\partial_{x}\left(\tilde{\lambda}_{i} v_{i}^{\varepsilon}\right)-\varepsilon \partial_{x x} v_{i}^{\varepsilon}=\phi_{i}^{\varepsilon}, \quad i=1, \cdots, N .
$$

Then, as the scalar case, we obtain that, for all $t \geq \tau_{\varepsilon}$,

$$
\left\|v_{i}^{\varepsilon}(t, \cdot)\right\|_{L^{1}} \leq\left\|v_{i}^{\varepsilon}\left(\tau_{\varepsilon}, \cdot\right)\right\|_{L^{1}}+\int_{\tau_{\varepsilon}}^{\infty} \int_{-\infty}^{\infty}\left|\phi_{i}^{\varepsilon}(t, x)\right| d x d t .
$$

(iv) Construct the basis $\left\{\mathbf{r}_{1}, \cdots, \mathbf{r}_{N}\right\}$ in an appropriate way so that, for $t \geq \tau_{\varepsilon}$,

$$
\int_{\tau_{\varepsilon}}^{\infty} \int_{-\infty}^{\infty}\left|\phi_{i}^{\varepsilon}(t, x)\right| d x d t \leq \hat{C}
$$

which implies

$$
\text { Tot.Var. }\left\{U^{\varepsilon}(t, \cdot)\right\}=\left\|U_{x}^{\varepsilon}(t, \cdot)\right\|_{L^{1}} \leq \sum_{i}\left\|v_{i}^{\varepsilon}(t, \cdot)\right\|_{L^{1}} \leq C,
$$

where $\hat{C}$ and $C$ are independent of $\varepsilon>0$.

Remark 1. The results above still hold even for non-conservative strictly hyperbolic systems. On the other hand, this approach requires both the artificial viscosity (i.e. $D(U)=I_{N \times N}$ ) and the total variation of the initial data sufficiently small.

Remark 2. A longstanding open problem is the $B V$-estimate and convergence of vanishing viscosity approximation $U^{\varepsilon}$ governed by the general form:

$$
\partial_{t} U^{\varepsilon}+\partial_{x} F\left(U^{\varepsilon}\right)=\varepsilon \partial_{x}\left(D\left(U^{\varepsilon}\right) \partial_{x} U^{\varepsilon}\right)
$$


for general viscosity matrices $D(U)$, including the Navier-Stokes viscosity matrices. This especially includes the fundamental problem in mathematical fluid dynamics, the inviscid limit of solutions of the NavierStokes equations to the Euler equations for homentropic flow, via the BV-estimate, which is still open.

\subsection{Compactness and Convergence via Compensated Compactness}

We now discuss the compactness and convergence of exact/approximate solutions to conservation laws via compensated compactness and related ideas, which only require much weak bounds that may be obtained easily through natural energy/entropy estimates as our examples below indicate.

\subsubsection{Scalar conservation laws}

Consider the Cauchy problem for scalar conservation laws (2.3) $(N=1)$ with initial data:

$$
\left.U\right|_{t=0}=U_{0} \in L^{\infty}(\mathbb{R}) .
$$

Then it can be easily shown that there exists $C$, independent of $\varepsilon$, such that the viscous solutions $U^{\varepsilon}$ satisfy the following natural estimates:

(i) Maximum principle: $\left\|U^{\varepsilon}\right\|_{L^{\infty}} \leq C$ or $\left\|U^{\varepsilon}\right\|_{L^{p}} \leq C$;

(ii) Dissipation estimate: $\left\|\sqrt{\varepsilon} U_{x}^{\varepsilon}\right\|_{L_{l o c}^{2}} \leq C$.

The second estimate is a direct corollary of the natural energy estimate:

$$
\varepsilon\left|\partial_{x} U^{\varepsilon}\right|^{2}=-\partial_{t}\left(\frac{\left|U^{\varepsilon}\right|^{2}}{2}\right)-\partial_{x}\left(\int^{U^{\varepsilon}} w F^{\prime}(w) d w\right)+\varepsilon \partial_{x x}\left(\frac{\left|U^{\varepsilon}\right|^{2}}{2}\right) .
$$

These estimates imply that, for any $\eta \in C^{2}$ with entropy flux $q(U)=$ $\int^{U} \eta^{\prime}(w) F^{\prime}(w) d w$

$$
\partial_{t} \eta\left(U^{\varepsilon}\right)+\partial_{x} q\left(U^{\varepsilon}\right) \text { is compact in } H_{l o c}^{-1} .
$$

Then the compensated compactness arguments yield the weak continuity of $F\left(U^{\varepsilon}\right)$, or even the strong convergence of $U^{\varepsilon}(t, x)$ a.e.

For the convex case, Tartar [83] was the first to employ one entropy pair $\left(\eta_{*}(U), q_{*}(U)\right)=\left(U^{2}, 2 \int^{U} w F^{\prime}(w) d w\right)$ to conclude the strong convergence, which initiated the successful applications of compensated compactness to nonlinear hyperbolic conservation laws. For the nonconvex case, the entropy pair $\left(\eta_{*}(U), q_{*}(U)\right)=\left(F(U), \int^{U}\left(F^{\prime}(w)\right)^{2} d w\right)$ 
also suffices to conclude the weak continuity with respect to the general equation, and the strong convergence when the equation is genuinely nonlinear for almost all $U$, as observed by Chen-Lu [17] and Luc Tartar independently. Also see Schonbek 78, DiPerna [34, and TadmorRascle-Bagnerini 82 .

The approach also applies to equation (2.6) $(N=1)$ with more general viscosity terms, as well as scalar conservation laws with memory [26].

For these, the following Murat-Tartar's div-curl lemma plays an essential role:

Div-Curl Lemma (Tartar [83, Murat 68]): Let $\Omega \subset \mathbb{R}^{d}, d \geq 2$, be open bounded. Let $p, q>1$ such that $\frac{1}{p}+\frac{1}{q}=1$. Assume that, for any $\varepsilon>0$, two vector fields

$$
u^{\varepsilon} \in L^{p}\left(\Omega ; \mathbb{R}^{d}\right), \quad v^{\varepsilon} \in L^{q}\left(\Omega ; \mathbb{R}^{d}\right)
$$

satisfy the following:

(i) $u^{\varepsilon} \rightarrow u$ weakly in $L^{p}\left(\Omega ; \mathbb{R}^{d}\right)$ as $\varepsilon \rightarrow 0$;

(ii) $v^{\varepsilon} \rightarrow v$ weakly in $L^{q}\left(\Omega ; \mathbb{R}^{d}\right)$ as $\varepsilon \rightarrow 0$;

(iii) $\operatorname{div} u^{\varepsilon}$ are confined in a compact subset of $W_{l o c}^{-1, p}(\Omega ; \mathbb{R})$;

(iv) $\operatorname{curl} v^{\varepsilon}$ are confined in a compact subset of $W_{l o c}^{-1, q}\left(\Omega ; \mathbb{R}^{d \times d}\right)$.

Then the scalar product of $u^{\varepsilon}$ and $v^{\varepsilon}$ are weakly continuous:

$$
u^{\varepsilon} \cdot v^{\varepsilon} \longrightarrow u \cdot v
$$

in the sense of distributions.

Various variations of this lemma for different applications/purposes have been developed; see Tartar [89, Briane, Casado-Diaz and Murat [6], and the references cited therein.

\subsubsection{Hyperbolic systems of conservation laws: Compensated compactness for the artificial viscosity method}

Consider system (2.5) with artificial viscosity. Assume that there exists a strictly convex entropy function $\eta_{*}(U), \nabla^{2} \eta_{*}(U)>0$. In many cases, it can be shown that there exists $C$ independent of $\varepsilon$ such that

(i) Invariant regions: $\left\|U^{\varepsilon}\right\|_{L^{\infty}} \leq C$;

(ii) Dissipation estimate: $\left\|\sqrt{\varepsilon} \partial_{x} U^{\varepsilon}\right\|_{L_{\text {loc }}^{2}} \leq C$. 
In fact, the dissipation estimate is natural as the scalar case, directly from the energy estimate as follows:

$$
\varepsilon\left(\partial_{x} U^{\varepsilon}\right)^{\top} \nabla^{2} \eta_{*}\left(U^{\varepsilon}\right) \partial_{x} U^{\varepsilon}=-\partial_{t} \eta_{*}\left(U^{\varepsilon}\right)-\partial_{x} q_{*}\left(U^{\varepsilon}\right)+\varepsilon \partial_{x x} \eta_{*}\left(U^{\varepsilon}\right) .
$$

Then, for any $\eta \in C^{2}$ with entropy flux $q$, i.e., $\nabla q(U)=\nabla \eta(U) \nabla F(U)$,

$$
\partial_{t} \eta\left(U^{\varepsilon}\right)+\partial_{x} q\left(U^{\varepsilon}\right) \text { is compact in } H_{l o c}^{-1} \text {. }
$$

The compensated compactness arguments can yield the strong convergence of $U^{\varepsilon}(t, x)$ when the system has strong nonlinearity.

The similar compensated compactness arguments apply to the systems with more general viscosity matrices (2.6) for $\nabla^{2} \eta_{*}(U) D(U) \geq$ $c_{0}>0$. Another advantage of this approach is to allow the initial data of large oscillation without bounded variation.

In order to achieve the strong compactness, as first indicated by Tartar [83, combining the div-curl lemma (83, 68]) and the Young measure representation theorem ( $c f$. Tartar [83]; also see 1, 3]), we have the following commutation identity for the associated Young measure $\nu=\nu_{(t, x)}(\boldsymbol{\lambda})$ (probability measure) for the sequence $U^{\varepsilon}(t, x)$ :

$$
\begin{aligned}
& \left\langle\nu(\boldsymbol{\lambda}), \eta_{1}(\boldsymbol{\lambda}) q_{2}(\boldsymbol{\lambda})-q_{1}(\boldsymbol{\lambda}) \eta_{2}(\boldsymbol{\lambda})\right\rangle \\
& =\left\langle\nu(\boldsymbol{\lambda}), \eta_{1}(\boldsymbol{\lambda})\right\rangle\left\langle\nu(\boldsymbol{\lambda}), q_{2}(\boldsymbol{\lambda})\right\rangle-\left\langle\nu(\boldsymbol{\lambda}), q_{1}(\boldsymbol{\lambda})\right\rangle\left\langle\nu(\boldsymbol{\lambda}), \eta_{2}(\boldsymbol{\lambda})\right\rangle
\end{aligned}
$$

for any entropy pairs $\left(\eta_{j}, q_{j}\right), j=1,2$, and

$$
\partial_{t}\langle\nu, \eta(\boldsymbol{\lambda})\rangle+\partial_{x}\langle\nu, q(\boldsymbol{\lambda})\rangle \leq 0
$$

in the sense of distributions for any convex entropy pair $(\eta, q), \nabla^{2} \eta \geq 0$. Then the main mathematical issue is whether $\nu$ is a Dirac measure. The key point is the imbalance of regularity of the operator in the commutation identity: The operator on the left is more regular than the one on the right due to cancellation when the system has strong nonlinearity. If so, the compactness of $U^{\varepsilon}(t, x)$ in $L^{1}$ follows.

For strict hyperbolicity with $N=2$, there are two families of entropy pairs determined by two arbitrary functions, which yield an affirmative answer to the issue; see DiPerna [33, Dafermos [27], Serre [9], Morawetz 66], Perthame-Tzavaras [75], and Chen-Li-Li [16].

Further challenges include nonstrictly hyperbolic systems, viscosity matrices with $\nabla^{2} \eta_{*}(U) D(U) \geq 0$ but not positive definite, and initial data of large oscillation with only energy bounds (without bounded variation or $L^{\infty}$-uniform bound). We now start with a fundamental example of nonstrictly hyperbolic systems. 


\subsubsection{Homentropic Euler equations: Compensated compact- ness for the artificial viscosity method}

The homentropic Euler equations take the following form:

$$
\left\{\begin{array}{l}
\partial_{t} \rho+\partial_{x} m=0, \\
\partial_{t} m+\partial_{x}\left(\frac{m^{2}}{\rho}+p(\rho)\right)=0,
\end{array}\right.
$$

where $\rho$ is the density, $u=\frac{m}{\rho}$ is the fluid velocity that is well-defined when $\rho>0, p=p(\rho)=\rho^{2} e^{\prime}(\rho)$ is the pressure with internal energy $e(\rho)$,

For a polytropic perfect gas,

$$
p(\rho)=\kappa \rho^{\gamma}, e(\rho)=\frac{\kappa}{\gamma-1} \rho^{\gamma-1},
$$

where $\gamma>1$ is the adiabatic exponent, and constant $\kappa$ in the pressuredensity relation may be chosen as $\kappa=\frac{(\gamma-1)^{2}}{4 \gamma}$ without loss of generality.

One of the main difficulties for solving (2.8) is that strict hyperbolicity fails when $\rho \rightarrow 0$.

An entropy function $\eta(\rho, m)$ is called a weak entropy if $\left.\eta(\rho, m)\right|_{\rho=0}=$ 0 . The weak entropy pairs can be represented as

$$
\eta^{\psi}(\rho, \rho u)=\int_{\mathbb{R}} \chi(s) \psi(s) d s, \quad q^{\psi}(\rho, \rho u)=\int_{\mathbb{R}}(\theta s+(1-\theta) u) \chi(s) \psi(s) d s
$$

for any $C^{2}$-test function $\psi(s)$, where $\chi(s)$ is the weak entropy kernel:

$$
\chi(s):=\left[\rho^{2 \theta}-(u-s)^{2}\right]_{+}^{\lambda}, \quad \theta=\frac{\gamma-1}{2}, \lambda=\frac{3-\gamma}{2(\gamma-1)} .
$$

The mechanical energy-energy flux pair $\left(\eta_{*}, q_{*}\right)$ :

$$
\eta_{*}(\rho, m)=\frac{1}{2} \frac{m^{2}}{\rho}+\rho e(\rho), \quad q_{*}(\rho, m)=\frac{1}{2} \frac{m^{3}}{\rho^{2}}+m\left(e(\rho)+\frac{p}{\rho}\right)
$$

is a convex entropy pair for (2.8).

Consider the homentropic Euler equations with artificial viscosity:

$$
\left\{\begin{array}{l}
\partial_{t} \rho+\partial_{x} m=\varepsilon \partial_{x}^{2} \rho, \\
\partial_{t} m+\partial_{x}\left(\frac{m^{2}}{\rho}+p(\rho)\right)=\varepsilon \partial_{x}^{2} m .
\end{array}\right.
$$

It can be shown for system (2.12) that there exists $C>0$, independent of $\varepsilon>0$, such that

(i) Invariant regions for the $L^{\infty}$-estimate:

$$
0 \leq \rho^{\varepsilon}(t, x) \leq C, \quad\left|m^{\varepsilon}(t, x)\right| \leq C \rho^{\varepsilon}(t, x) \quad \text { a.e. }
$$


(ii) Dissipation estimate:

$$
\sqrt{\varepsilon}\left\|\partial_{x}\left(\rho^{\varepsilon}, m^{\varepsilon}\right)\right\|_{L^{2}([0, T] \times \mathbb{R})} \leq C,
$$

via the mechanical energy pair $\left(\eta_{*}, q_{*}\right)$ that is strictly convex for $1<\gamma \leq 2$, and convex for $\gamma>2$ for which a corresponding weighted dissipation estimate can be obtained.

These estimates yield that, for any $C^{2}$ weak entropy pair $(\eta, q)$,

$$
\partial_{t} \eta\left(\rho^{\varepsilon}, m^{\varepsilon}\right)+\partial_{x} q\left(\rho^{\varepsilon}, m^{\varepsilon}\right) \text { is compact in } H_{l o c}^{-1} .
$$

Then the convergence problem for $\left(\rho^{\varepsilon}, m^{\varepsilon}\right)$ is reduced to the reduction problem for a measure-valued solution $\nu_{t, x}$ :

If $\operatorname{supp} \nu_{t, x}$ is bounded, then

$$
\nu_{t, x}=\nu_{(\rho(t, x), m(t, x))},
$$

that is, $\left(\rho^{\varepsilon}(t, x), m^{\varepsilon}(t, x)\right) \rightarrow(\rho(t, x), m(t, x))$ a.e. $(t, x)$.

This problem has been solved by DiPerna [32] for $\gamma=\frac{N+2}{N}, N \geq 5$ odd, Ding-Chen-Luo [30] and Chen [8] for $\gamma \in\left(1, \frac{5}{3}\right]$, Lions-PerthameTadmor [62] for $\gamma \geq 3$, Lions-Perthame-Souganidis 61] for $\gamma \in\left(\frac{5}{3}, 3\right)$, and Chen-LeFloch 14 for general pressure laws. The key point is to employ effectively the weak entropy pairs in the commutation identity (2.7) for the associated Young measure $\nu_{t, x}$ with compact support.

The convergence of related numerical methods with corresponding numerical viscosity matrices including the Lax-Friedrichs scheme and Godunov scheme has also been established in Ding-Chen-Luo [30; also see Chen [10].

The isothermal case $\gamma=1$ has also been handled by Huang-Wang [50]; also see LeFloch-Shelukhin [57].

Some further important problems include the inviscid limit from the compressible Navier-Stokes equations to the compressible Euler equations (see §2.3) and the existence of global spherically symmetric solutions to the compressible Euler equations (see $\S 2.4$ ).

\subsection{Navier-Stokes Equations: Inviscid Limit}

Consider the Cauchy problem:

$$
\left\{\begin{array}{l}
\rho_{t}+(\rho u)_{x}=0, \\
(\rho u)_{t}+\left(\rho u^{2}+p\right)_{x}=\varepsilon u_{x x},
\end{array}\right.
$$

with the initial conditions:

$$
\left.(\rho, u)\right|_{t=0}=\left(\rho_{0}^{\varepsilon}(x), u_{0}^{\varepsilon}(x)\right), \quad \lim _{x \rightarrow \pm \infty}\left(\rho_{0}^{\varepsilon}(x), u_{0}^{\varepsilon}(x)\right)=\left(\rho^{ \pm}, u^{ \pm}\right),
$$


where $\left(\rho^{ \pm}, u^{ \pm}\right)$are constant end-states with $\rho^{ \pm}>0$, and the viscosity coefficient $\varepsilon \in\left(0, \varepsilon_{0}\right]$ for some fixed $\varepsilon_{0}$.

The existence of $C^{2}$-solutions $\left(\rho^{\varepsilon}, u^{\varepsilon}\right)(t, x)$ for large initial data was obtained by Kanel 52 for the same ending states and by Hoff [46] for different ending states.

Inviscid Limit Problem: Does the solution sequence $\left(\rho^{\varepsilon}, u^{\varepsilon}\right)(t, x)$ of system (2.14) strongly converge to a solution to the homentropic Euler equations (2.8) when $\varepsilon \rightarrow 0$ ?

This problem has been addressed by Gilbarg [38, Hoff-Liu [47, and Gùes-Métivier-Williams-Zumbrun 43 for some physical cases with special structure for which the limit solution contains only one shock.

For the general case, several new difficulties arise, which include

(i) No invariant regions: Only energy norms;

(ii) Direct derivative estimates only partially: $\left\|\sqrt{\varepsilon} \partial_{x} u^{\varepsilon}\right\|_{L_{l o c}^{2}} \leq C$;

(iii) No a priori bounded support of the measure-valued solution $\nu_{t, x}$.

Nevertheless, the following theorem has been established.

Theorem (Chen-Perepelitsa [18]): Let the initial functions $\left(\rho_{0}^{\varepsilon}, u_{0}^{\varepsilon}\right)$ satisfy

$$
\begin{aligned}
& \int_{-\infty}^{\infty} \Phi_{*}\left(\rho_{0}^{\varepsilon}(x), m_{0}^{\varepsilon}(x)\right) d x \leq E_{0}<\infty \\
& \int_{-\infty}^{\infty}\left(\varepsilon^{2} \frac{\left|\rho_{0, x}^{\varepsilon}(x)\right|^{2}}{\rho_{0}^{\varepsilon}(x)^{3}}+2 \varepsilon \frac{\left|\rho_{0, x}^{\varepsilon}(x) u_{0}^{\varepsilon}(x)\right|}{\rho_{0}^{\varepsilon}(x)}+\rho_{0}^{\varepsilon}(x)\left|u_{0}^{\varepsilon}(x)\right|\right) d x \leq E_{1}<\infty
\end{aligned}
$$

where $\Phi_{*}(\rho, m)=\eta_{*}(\rho, m)-\eta_{*}(\bar{\rho}, \bar{m})-\nabla \eta_{*}(\bar{\rho}, \bar{m}) \cdot(\rho-\bar{\rho}, m-\bar{m}) \geq 0$ for $\bar{m}=\bar{\rho} \bar{u},(\bar{\rho}, \bar{u})$ is a pair of smooth monotone functions satisfying $(\bar{\rho}(x), \bar{u}(x))=\left(\rho^{ \pm}, u^{ \pm}\right)$when $\pm x \geq L_{0}$ for some large $L_{0}>0$, and both $E_{0}$ and $E_{1}$ are independent of $\varepsilon$. Let $\left(\rho^{\varepsilon}, m^{\varepsilon}\right), m^{\varepsilon}=\rho^{\varepsilon} u^{\varepsilon}$, be the solution of the Cauchy problem for the Navier-Stokes equations (2.14) for each fixed $\varepsilon>0$. Then, when $\varepsilon \rightarrow 0$, there exists a subsequence of $\left(\rho^{\varepsilon}, m^{\varepsilon}\right)$ that converges strongly almost everywhere to a finite-energy solution $(\rho, m)$ to the Cauchy problem for the homentropic Euler equations (2.8) for any $\gamma>1$.

The strategies for this include the following steps.

(i) Derive the finite-energy bound and higher integrability bound (replacing $L^{\infty}$ bound);

(ii) Derive new derivative estimate for $\varepsilon \partial_{x} \rho^{\varepsilon}$; 
(iii) Show the $H^{-1}$-compactness of weak entropy dissipation measures only for weak entropy pairs with compactly supported $C^{2}$-test functions;

(iv) Prove that any connected component of support of the measurevalued solution $\nu_{t, x}$ must be bounded, which reduces to the case when the support of $\nu_{t, x}$ is bounded as in $\S 2.2 .3$.

To achieve these, the following key estimates of solutions to the Navier-Stokes equations are essential: There exist $C_{1}>0$ and $C_{2}=$ $C_{2}\left(E_{0}, E_{1}, K, \gamma, t\right)$ independent of $\varepsilon$ for any compact set $K \subset \mathbb{R}$ such that, for any $t>0$,

(i) Energy estimate:

$$
\int_{-\infty}^{\infty} \Phi_{*}\left(\rho^{\varepsilon}(t, x), m^{\varepsilon}(t, x)\right) d x+\int_{0}^{t} \int_{-\infty}^{\infty} \varepsilon\left|u_{x}^{\varepsilon}\right|^{2} d x d \tau \leq E_{0}
$$

(ii) New derivative estimate for the density:

$$
\varepsilon^{2} \int \frac{\left|\rho_{x}^{\varepsilon}(t, x)\right|^{2}}{\rho^{\varepsilon}(t, x)^{3}} d x+\varepsilon \int_{0}^{t} \int_{-\infty}^{\infty}\left(\rho^{\varepsilon}\right)^{\gamma-3}\left|\rho_{x}^{\varepsilon}\right|^{2} d x d \tau \leq C_{1}\left(E_{0}+E_{1}\right) .
$$

(iii) Higher integrability bound:

$$
\int_{0}^{t} \int_{K}\left(\rho^{\varepsilon}\left|u^{\varepsilon}\right|^{3}+\left(\rho^{\varepsilon}\right)^{\gamma+\theta}+\left(\rho^{\varepsilon}\right)^{\gamma+1}\right) d x d \tau \leq C_{2} .
$$

The higher integrability estimate (iii) is motivated by the related work by Lions-Perthame-Tadmor $[62$ and LeFloch-Westdickenberg [58]. For some related earlier work on the convergence of approximate solutions in the $L^{p}$-framework, see Serre-Shearer [80] for a $2 \times 2$ system of elasticity with severe growth conditions, and LeFloch-Westdickenberg 58. for the convergence of approximate solutions with full dissipation in the energy norms for the homentropic Euler equations with $\gamma \in\left(1, \frac{5}{3}\right]$.

Let $\nu_{t, x}$ be the Young measure determined by the solutions of the Navier-Stokes equations (2.14). Then $\nu_{t, x}$ is confined by

$$
\begin{aligned}
& \theta\left(s_{2}-s_{1}\right)\left(\overline{\chi\left(s_{1}\right) \chi\left(s_{2}\right)}-\overline{\chi\left(s_{1}\right)} \overline{\chi\left(s_{2}\right)}\right) \\
& =(1-\theta)\left(\overline{u \chi\left(s_{2}\right)} \overline{\chi\left(s_{1}\right)}-\overline{u \chi\left(s_{1}\right)} \overline{\chi\left(s_{2}\right)}\right) \quad \text { for a.e. } s_{1}, s_{2} \in \mathbb{R}
\end{aligned}
$$

for the entropy kernel $\chi(s):=\left[\rho^{2 \theta}-(u-s)^{2}\right]_{+}^{\lambda}$ with $\theta=\frac{\gamma-1}{2}$ and $\lambda=\frac{3-\gamma}{2(\gamma-1)}$, where $\overline{f(s)}:=\left\langle\nu_{t, x}, f(s ; \rho, u)\right\rangle$. 
The goal is to establish that the Young measure is a Dirac mass in the phase plane for $(\rho, m)$. The new difficulty is now that supp $\nu_{t, x}$ is unbounded in general.

We divide the proof into three cases:

Case 1: $\gamma=3$. The same argument for the bounded support of $\nu_{t, x}$ applies as in 62. In this case, $\theta=1$ and the commutation relation becomes

$$
\overline{\chi\left(s_{1}\right) \chi\left(s_{2}\right)}=\overline{\chi\left(s_{1}\right)} \overline{\chi\left(s_{2}\right)},
$$

which implies $\overline{\chi(s)}^{2}=\overline{\chi(s)^{2}}$ by taking $s_{1}=s_{2}$. That is,

$$
\left\langle\nu_{t, x},(\chi(s)-\overline{\chi(s)})^{2}\right\rangle=0 \quad \text { for any } s \in \mathbb{R} .
$$

This implies that $\nu$ must be a Dirac mass on the set $\{\rho>0\}$ or be supported completely in the vacuum $V=\{\rho=0\}$, that is, the measurevalued solution $\nu_{t, x}$ is a Dirac mass (2.13) in the phase plane for $(\rho, m)$.

Case 2: $\gamma>3$. Let $A:=\cup\left\{\left(u-\rho^{\theta}, \rho^{\theta}+u\right):(\rho, u) \in \operatorname{supp} \nu\right\}$. Let $J=\left(s_{-}, s_{+}\right)$be any connected component of $A$. Note that $\operatorname{supp} \chi(s)=$ $\left\{(\rho, u): u-\rho^{\theta} \leq s \leq u+\rho^{\theta}\right\}$.

Claim: The connected component $J$ is bounded for $\gamma>3$.

On the contrary, let $\inf \{s: s \in J\}=-\infty$. Our strategy is to fix $M_{0}$ first such that $M_{0}+1 \in J$ and restrict $s_{2} \in\left(M_{0}, M_{0}+1\right)$, and then choose sufficiently small $s_{1} \leq-2\left|M_{0}\right|$ to reach the contradiction.

To achieve this, two following estimates are essential:

(i) $\int_{M_{0}}^{M_{0}+1} \frac{\overline{\chi\left(s_{1}\right) \chi\left(s_{2}\right)}}{\overline{\chi\left(s_{1}\right)}} d s_{2} \leq C(\lambda)\left|s_{1}\right|^{\lambda}$ for $\lambda<0$, which is our key new observation.

(ii) $\frac{\overline{\chi\left(s_{1}\right) \chi\left(s_{2}\right)}}{\overline{\chi\left(s_{1}\right)}} \geq \overline{\chi\left(s_{2}\right)}$ a.e. $s_{1}, s_{2} \in J, s_{1}<s_{2}$, by employing LionsPerthame-Tadmor's argument in 62.

Combining the two estimates, we have

$$
\int_{M_{0}}^{M_{0}+1} \frac{\overline{\chi\left(s_{1}\right) \chi\left(s_{2}\right)}}{\overline{\chi\left(s_{1}\right)}} d s_{2} \geq \int_{M_{0}}^{M_{0}+1} \overline{\chi\left(s_{2}\right)} d s_{2}=C\left(M_{0}, \lambda\right)>0,
$$

which implies that, when $s_{1} \rightarrow-\infty$,

$$
0<C\left(M_{0}, \lambda\right)=\int_{M_{0}}^{M_{0}+1} \frac{\overline{\chi\left(s_{1}\right) \chi\left(s_{2}\right)}}{\overline{\chi\left(s_{1}\right)}} d s_{2} \leq C(\lambda)\left|s_{1}\right|^{\lambda} \rightarrow 0 .
$$

This arrives at the contradiction.

The case when $J$ is unbounded from above can be treated similarly. 
This indicates that any connected component $J$ of the support of the Young measure $\nu$ is bounded for $\gamma>3$, which reduces to the LionsPerthame-Tadmor's case for $\gamma>3$ in 62.

Case 3: $\gamma \in(1,3)$. On the contrary, suppose that a connected component $J$ of the support is unbounded from below.

Let $M_{0}=\sup \{s: s \in J\} \in(-\infty, \infty]$. Let $s_{1}, s_{2}, s_{3} \in\left(-\infty, M_{0}\right)$ with $s_{1}<s_{2}<s_{3}$. The commutation relation leads to

$$
\begin{aligned}
& \left(s_{2}-s_{1}\right) \frac{\overline{\chi\left(s_{1}\right) \chi\left(s_{2}\right)}}{\overline{\chi\left(s_{1}\right)}}+\left(s_{3}-s_{2}\right) \frac{\overline{\chi\left(s_{3}\right) \chi\left(s_{2}\right)}}{\overline{\chi\left(s_{3}\right)}} \\
& =\left(s_{3}-s_{1}\right) \overline{\chi\left(s_{2}\right)} \frac{\overline{\chi\left(s_{1}\right) \chi\left(s_{3}\right)}}{\overline{\chi\left(s_{1}\right)} \overline{\chi\left(s_{3}\right)}} .
\end{aligned}
$$

Differentiating this equation in $s_{2}$ and dividing by $\left(s_{3}-s_{1}\right)$, we obtain

$$
\begin{aligned}
\overline{\chi^{\prime}\left(s_{2}\right)} \frac{\overline{\chi\left(s_{1}\right) \chi\left(s_{3}\right)}}{\overline{\chi\left(s_{1}\right)} \overline{\chi\left(s_{3}\right)}}= & \frac{s_{2}-s_{1}}{s_{3}-s_{1}} \frac{\overline{\chi\left(s_{1}\right) \chi^{\prime}\left(s_{2}\right)}}{\overline{\chi\left(s_{1}\right)}}+\frac{s_{3}-s_{2}}{s_{3}-s_{1}} \frac{\overline{\chi\left(s_{3}\right) \chi^{\prime}\left(s_{2}\right)}}{\overline{\chi\left(s_{3}\right)}} \\
& +\frac{1}{s_{3}-s_{1}} \frac{\overline{\chi\left(s_{1}\right) \chi\left(s_{2}\right)}}{\overline{\chi\left(s_{1}\right)}}-\frac{1}{s_{3}-s_{1}} \frac{\overline{\chi\left(s_{3}\right) \chi\left(s_{2}\right)}}{\overline{\chi\left(s_{3}\right)}} .
\end{aligned}
$$

Our strategy is to take $s_{1} \rightarrow-\infty$ first and show then that the lefthand side has a smaller order than the right-hand side to arrive at the contradiction.

To do this, we divide the argument into five steps:

(i). Show the estimate:

$$
\frac{\overline{\chi\left(s_{1}\right) \chi\left(s_{3}\right)}}{\overline{\overline{\chi\left(s_{1}\right)}} \overline{\chi\left(s_{3}\right)}} \geq 1 \quad \text { for any } s_{1}, s_{3} \in J
$$

by employing Lions-Perthame-Tadmor's argument in 662.

(ii). Show that $\overline{\chi(s)} \geq 0$, but is not identically zero, and $\overline{\chi(s)} \rightarrow 0$ as $s \rightarrow \inf J, \sup J$. This yields that there exists $s_{2}$ such that $\overline{\chi^{\prime}\left(s_{2}\right)}>0$ and $\overline{\chi\left(s_{2}\right)}>0$.

(iii). Let $s_{3}>s_{2}$ be the points such that $\overline{\chi\left(s_{3}\right)}>0$, and let $s_{1} \rightarrow-\infty$. From the first identity (2.16),

$$
\frac{\overline{\chi\left(s_{1}\right) \chi\left(s_{2}\right)}}{\overline{\chi\left(s_{1}\right)}}=\overline{\chi\left(s_{2}\right)} \frac{\overline{\chi\left(s_{1}\right) \chi\left(s_{3}\right)}}{\overline{\chi\left(s_{1}\right)} \overline{\chi\left(s_{3}\right)}}+o(1) \quad \text { as } s_{1} \rightarrow-\infty \text {. }
$$

(iv). Show that $\left[\chi^{\prime}(s)\right]_{+} \leq \frac{2 \lambda}{s-s_{1}} \chi(s)$. 
(v). From the second equation (2.17), by throwing away the negative terms, we have

$$
\overline{\chi^{\prime}\left(s_{2}\right)} \frac{\overline{\chi\left(s_{1}\right) \chi\left(s_{3}\right)}}{\overline{\chi\left(s_{1}\right)} \overline{\chi\left(s_{3}\right)}} \leq \frac{2 \lambda+1}{s_{3}-s_{1}} \frac{\overline{\chi\left(s_{1}\right) \chi\left(s_{2}\right)}}{\overline{\chi\left(s_{1}\right)}}+o(1),
$$

which implies

$$
\left(\overline{\chi^{\prime}\left(s_{2}\right)}-\frac{2 \lambda+1}{s_{3}-s_{1}} \overline{\chi\left(s_{2}\right)}\right) \frac{\overline{\chi\left(s_{1}\right) \chi\left(s_{3}\right)}}{\overline{\chi\left(s_{1}\right)} \overline{\chi\left(s_{3}\right)}} \leq o(1) .
$$

This arrives at the contradiction as $s_{1} \rightarrow-\infty$.

Another different proof is given by LeFloch-Westdickenberg [58] for $1<\gamma \leq \frac{5}{3}$. The inviscid limit of the viscous shallow water equations to the Saint-Venant system has also been established in Chen-Perepelitsa [19.

\subsection{Spherically Symmetric Solutions to the Multidi- mensional Homentropic Euler Equations}

The homentropic Euler equations for multidimensional compressible fluids take the following form:

$$
\left\{\begin{array}{l}
\rho_{t}+\nabla_{\mathbf{x}}(\rho \mathbf{v})=0, \\
(\rho \mathbf{v})_{t}+\nabla_{\mathbf{x}}(\rho \mathbf{v} \otimes \mathbf{v})+\nabla_{\mathbf{x}} p=0,
\end{array}\right.
$$

where $\mathbf{x}=\left(x_{1}, \ldots, x_{d}\right) \in \mathbb{R}^{d}, \nabla_{\mathbf{x}}$ is the gradient with respect to $\mathbf{x} \in$ $\mathbb{R}^{d}$, and $\mathbf{v}=\left(v_{1}, \ldots, v_{d}\right) \in \mathbb{R}^{d}$ is the velocity. The pressure-density constitutive relation (by scaling) satisfies (2.9).

We seek the spherically symmetric solutions with form:

$$
\rho(t, \mathbf{x})=\rho(t, r), \quad \mathbf{v}(t, \mathbf{x})=u(t, r) \frac{\mathbf{x}}{r}, \quad r=|\mathbf{x}| .
$$

Then the functions $(\rho, m)=(\rho, \rho u)$ are governed by

$$
\left\{\begin{array}{l}
\rho_{t}+m_{r}+\frac{d-1}{r} m=0, \\
m_{t}+\left(\frac{m^{2}}{\rho}+p(\rho)\right)_{r}+\frac{d-1}{r} \frac{m^{2}}{\rho}=0 .
\end{array}\right.
$$

For the defocusing case, the existence of expanding spherically symmetric solutions with the following bounds:

$$
0 \leq \rho(t, r)^{\frac{\gamma-1}{2}} \leq u(t, r) \leq C<\infty,
$$

has been constructed, provided that the initial functions have the same bounds, in Chen $[9]$. 
For the focusing case, the singularity of imploding self-similar spherically symmetric solutions has been discussed in [25, 42, 77, 91. It is indicated indeed in Rauch [76] that there is no $B V$ or $L^{\infty}$ bound for the imploding solutions in general.

A longstanding open problem is whether the concentration phenomenon occurs at the origin, that is, whether the density $\rho$ develops a measure at the origin. In Chen-Perepelitsa [20, we have developed a method of vanishing artificial viscosity to prove that the vanishing viscosity limit solution does not form concentration at the origin, but has a bounded total energy. More precisely, we construct a sequence of vanishing viscosity solutions to the following initial-boundary problem:

$$
\left\{\begin{array}{l}
\rho_{t}+m_{r}+\frac{d-1}{r} m=\varepsilon\left(\rho_{r r}+\frac{d-1}{r} \rho_{r}\right), \\
m_{t}+\left(\frac{m^{2}}{\rho}+p_{\delta}(\rho)\right)_{r}+\frac{d-1}{r} \frac{m^{2}}{\rho}=\varepsilon\left(m_{r r}+\frac{d-1}{r} m\right)_{r},
\end{array}\right.
$$

with appropriate approximate initial data:

$$
\left.(\rho, m)\right|_{t=0}=\left(\rho_{0}^{\varepsilon}(r), m_{0}^{\varepsilon}(r)\right) \rightarrow\left(\rho_{0}, m_{0}\right) \quad \text { a.e. as } \varepsilon \rightarrow 0,
$$

and the boundary condition:

$$
\left.\left(\rho_{r}, m\right)\right|_{r=a(\varepsilon)}=(0,0),\left.\quad(\rho, m)\right|_{r=b(\varepsilon)}=(\bar{\rho}(\varepsilon), 0),
$$

where $\left(\rho_{0}, m_{0}\right)$ is the initial data for the spherical symmetric solution to system (2.20), $p_{\delta}(\rho)=\delta \rho^{2}+\kappa \rho^{\gamma}$ with $\delta=\delta(\varepsilon)$, and $a(\varepsilon), b(\varepsilon), \bar{\rho}(\varepsilon)$, and $\delta(\varepsilon)$ are positive with $a(\varepsilon) \rightarrow 0, b(\varepsilon) \rightarrow \infty,(\bar{\rho}(\varepsilon), \delta(\varepsilon)) \rightarrow(0,0)$, as well as certain combinations of $(b(\varepsilon), \bar{\rho}(\varepsilon), \delta(\varepsilon))$ tending to 0 , as $\varepsilon \rightarrow 0$ (cf. [20]). Then we have

Theorem (Chen-Perepelitsa 20]). Let the initial functions $\left(\rho_{0}, m_{0}\right)$ for system (2.20) satisfy the finite-energy conditions. Then

(i) For sufficiently small fixed $\varepsilon>0$, there exists a global viscous solution $\left(\rho^{\varepsilon}, m^{\varepsilon}\right)$ to the initial-boundary value problem (2.21)-(2.23) satisfying that, for any compact set $K \subset \mathbb{R}_{+}$and $T>0$, there exists $C_{T}>0$ independent of $\varepsilon>0$ such that, for any $0<t \leq T$,

$$
\begin{aligned}
& \int_{a(\varepsilon)}^{b(\varepsilon)}\left(\frac{1}{2} \rho^{\varepsilon}\left(u^{\varepsilon}\right)^{2}+\rho^{\varepsilon} e\left(\rho^{\varepsilon}\right)\right)(t, r) r^{d-1} d r \\
& \quad+\varepsilon \int_{0}^{t} \int_{a(\varepsilon)}^{b(\varepsilon)}\left(\left(\rho^{\varepsilon}\right)^{\gamma-2}\left|\rho_{r}^{\varepsilon}\right|^{2}+\rho^{\varepsilon}\left|u_{r}^{\varepsilon}\right|^{2}+\frac{\rho^{\varepsilon}\left(u^{\varepsilon}\right)^{2}}{2 r^{2}}\right) r^{d-1} d r d t \leq C_{T},
\end{aligned}
$$

and

$$
\int_{0}^{t} \int_{K}\left(\rho^{\varepsilon}\left|u^{\varepsilon}\right|^{3}+\left(\rho^{\varepsilon}\right)^{\gamma+\theta}+\left(\rho^{\varepsilon}\right)^{\gamma+1}\right) r^{d-1} d r d \tau \leq C_{T}
$$


(ii) When $\varepsilon \rightarrow 0$, there exists a subsequence of $\left(\rho^{\varepsilon}, m^{\varepsilon}\right)$ that converges strongly almost everywhere to a finite-energy spherically symmetric solution $(\rho, m)$ to system (2.20) for any $\gamma>1$ with initial data $\left(\rho_{0}, m_{0}\right)$.

The key ingredients are the uniform a priori estimates in (i) in combination with the reduction of the corresponding Young measure discussed in $\S 2.3$.

Recently, we have also solved and/or made progress on several fundamental problems in nonlinear partial differential equations by employing the viscosity method. These include vanishing viscosity approximation for transonic flow in Chen-Slemrod-Wang [21] (also see Morawetz [65, 67), and subsonic-sonic limit of exact/approximate solutions to the full Euler equations for multidimensional steady compressible fluids in Chen-Huang-Wang [13].

\section{Weak Continuity and Rigidity of the Gauss- Codazzi-Ricci System and Corresponding Isometric Embeddings}

The isometric embedding problem is a longstanding fundamental problem in differential geometry. As is well-known from differential geometry, given a surface, we can compute its metric $\left\{g_{i j}\right\}$ and associated first fundamental form:

$$
I=\sum g_{i j} d x^{i} d x^{j}
$$

and its curvatures determined by the second fundamental form:

$$
I I=\sum h_{i j} d x^{i} d x^{j} .
$$

Then a natural mathematical question is as follows:

Isometric Embedding Problem: Given a metric $\left\{g_{i j}\right\}$, can we find a surface in the Euclidean space with the given metric $\left\{g_{i j}\right\}$ ?

In other words, we seek a map $\mathbf{r}: \Omega \rightarrow \mathbb{R}^{N}$ such that

$$
d \mathbf{r} \cdot d \mathbf{r}=\sum_{i, j=1}^{N} g_{i j} d x^{i} d x^{j}
$$

in the local coordinates, that is, $\partial_{x^{i}} \mathbf{r} \cdot \partial_{x^{j}} \mathbf{r}=g_{i j}$ so that $\left(\partial_{x^{i}} \mathbf{r}, \partial_{x^{j}} \mathbf{r}\right), i \neq$ $j$, in $\mathbb{R}^{d}$ are linearly independent.

This is an inverse problem, which is a realization question for given an abstract metric $\left\{g_{i j}\right\}$. A further question is whether we can produce 
even more sophisticated surfaces or thin sheets for applications. These questions are truly fundamental, not only in mathematics such as differential geometry and topology, but also in many applications such as the understanding evolution of sophisticated shapes of surfaces or thin sheets in nature including elastic materials, protein folding in biology and algorithmic origami, as well as design, visual arts, among others.

The mathematical study of this problem has a long history, including the early important work by Schlaefli (1873), Darboux (1894), Hilbert (1901), Weyl (1916), Janet (1926-27), Cartan (1926-27); also see HanHong [45] and the references cited therein. In particular, Nash 73 ] established the Nash isometric embedding theorem (also called $C^{k}$-embedding theorem, $k \geq 3)$ :

Every $n$-dimensional Riemannian manifold (analytic or $C^{k}, k \geq 3$ ) can be $C^{k}$-isometrically imbedded in the Euclidean space $\mathbb{R}^{d}$ with $d=$ $2 s_{n}+4 n$ for the compact case and $d=(n+1)\left(3 s_{n}+4 n\right)$ for the noncompact case, where $s_{n}=\frac{n(n+1)}{2}$ is the Jenet dimension (cf. [51]).

The results were further improved with lowerer target dimensions by Gromov [41] with $d=s_{n}+2 n+3$ and Günther [44] with $d=\max \left\{s_{n}+\right.$ $\left.2 n, s_{n}+n+5\right\}$.

The following further problems are important for applications:

(i) Rigidity of isometric embeddings: Is a weak limit of a sequence of isometric embeddings in some topology still an isometric embedding?

(ii) Lowerest target dimension for global isometric embeddings, which is expected to be the Janet dimension $d=s_{n}$;

(iii) Optimal or assigned regularity such as $C^{1,1}, W^{2, p}$, and $B V^{1}$. The regularity issue is quite sensitive. For example, Efimov's example in 36 indicates that there is no $C^{2}$-isometric embedding when $n=2$ and $d=s_{n}=3$.

For $n=2$ and $d=3$, the fundamental theorem in differential geometry indicates that

There exists a surface in $\mathbb{R}^{3}$ whose first and second fundamental forms are $I$ and $I I$, if the coefficients $\left\{g_{i j}\right\}$ and $\left\{h_{i j}\right\}$ of the two given quadratic forms $I$ and II, I being positive definite, satisfy the GaussCodazzi system. That is, given $\left\{g_{i j}\right\}$, the second fundamental coefficients $\left\{h_{i j}\right\}$ are determined by the Codazzi equations (compatibility):

$$
\left\{\begin{array}{l}
\partial_{x} M-\partial_{y} L=L \Gamma_{22}^{(2)}-2 M \Gamma_{12}^{(2)}+N \Gamma_{11}^{(2)}, \\
\partial_{x} N-\partial_{y} M=-L \Gamma_{22}^{(1)}+2 M \Gamma_{12}^{(1)}-N \Gamma_{11}^{(1)}
\end{array}\right.
$$


subject to the Gauss equation (i.e., the Monge-Ampère type constraint):

$$
L N-M^{2}=K
$$

where

$$
L=\frac{h_{11}}{\sqrt{|g|}}, \quad M=\frac{h_{12}}{\sqrt{|g|}}, N=\frac{h_{22}}{\sqrt{|g|}},|g|=g_{11} g_{22}-g_{12}^{2},
$$

$\Gamma_{i j}^{(k)}$ are the Christoffel symbols, depending on $g_{i j}$ up to their first derivatives, and $K(x, y)$ is the Gauss curvature, determined by $g_{i j}$ up to their second derivatives.

This theorem holds even when $h_{i j} \in L^{p}(c f$. Maradare [63, 64]). Note that system (3.1) with (3.2) is a system of nonlinear PDEs of mixed elliptic-hyperbolic type, which is determined by the sign of the Gauss curvature $K$. Surfaces with Gauss curvature of changing sign are very normal in geometry, including tori such as toroidal shells or doughnut surfaces.

Fluid dynamics formalism for isometric embedding (ChenSlemrod-Wang [22]). Set $L=\rho v^{2}+p, M=-\rho u v, N=\rho u^{2}+p$, and $q^{2}=u^{2}+v^{2}$. Choose $p$ as the Chaplygin type gas: $p=-\frac{1}{\rho}$.

The Codazzi equations (3.1) become the balance laws of momentum equations:

$$
\left\{\begin{array}{l}
\partial_{x}(\rho u v)+\partial_{y}\left(\rho v^{2}+p\right)=-\left(\rho v^{2}+p\right) \Gamma_{22}^{(2)}-2 \rho u v \Gamma_{12}^{(2)}-\left(\rho u^{2}+p\right) \Gamma_{11}^{(2)}, \\
\partial_{x}\left(\rho u^{2}+p\right)+\partial_{y}(\rho u v)=-\left(\rho v^{2}+p\right) \Gamma_{22}^{(1)}-2 \rho u v \Gamma_{12}^{(1)}-\left(\rho u^{2}+p\right) \Gamma_{11}^{(1)},
\end{array}\right.
$$

and the Gauss equation becomes the Bernoulli relation:

$$
p=-\sqrt{q^{2}+K} .
$$

Define the sound speed: $c^{2}=p^{\prime}(\rho)$. Then $c^{2}=\frac{1}{\rho^{2}}=q^{2}+K$.

$c^{2}>q^{2}$ and the "flow" is subsonic when $K>0$;

$c^{2}<q^{2}$ and the "flow" is supersonic when $K<0$;

$c^{2}=q^{2}$ and the "flow" is sonic when $K=0$.

Based on this connection, the existence and continuity of isometric embeddings via compensated compactness and entropy analysis were first addressed in Chen-Slemrod-Wang [22].

For higher dimensional case, the isometric embeddings of $n$-dimensional Riemannian manifolds $(n \geq 3)$ into $\mathbb{R}^{d}$ are described by the following Gauss-Codazzi-Ricci system: 
Gauss equations:

$$
h_{j i}^{a} h_{k l}^{a}-h_{k i}^{a} h_{j l}^{a}=R_{i j k l}
$$

\section{Codazzi equations:}

$$
\frac{\partial h_{l j}^{a}}{\partial x^{k}}-\frac{\partial h_{k j}^{a}}{\partial x^{l}}+\Gamma_{l j}^{m} h_{k m}^{a}-\Gamma_{k j}^{m} h_{l m}^{a}+\kappa_{k b}^{a} h_{l j}^{b}-\kappa_{l b}^{a} h_{k j}^{b}=0 ;
$$

\section{Ricci equations:}

$$
\frac{\partial \kappa_{l b}^{a}}{\partial x^{k}}-\frac{\partial \kappa_{k b}^{a}}{\partial x^{l}}-g^{m n}\left(h_{m l}^{a} h_{k n}^{b}-h_{m k}^{a} h_{l n}^{b}\right)+\kappa_{k c}^{a} \kappa_{l b}^{c}-\kappa_{l c}^{a} \kappa_{k b}^{c}=0,
$$

where $\left\{R_{i j k l}\right\}$ is the Riemann curvature tensor, $\kappa_{k b}^{a}=-\kappa_{k a}^{b}$ are the coefficients of the connection form (torsion coefficients) on the normal bundle; the indices $a, b, c$ run from 1 to $N$, and $i, j, k, l, m, n$ run from 1 to $d \geq 3$.

The Gauss-Codazzi-Ricci system (3.3)-3.5) has no type, neither purely hyperbolic nor purely elliptic for general Riemann curvature tensor $R_{i j k l}$; see Bryant-Griffiths-Yang [7. Even though, we have established the following weak continuity and rigidity of system (3.3)-(3.5) and corresponding embedded surfaces:

Theorem (Chen-Slemrod-Wang [23]). Consider the Gauss-CodazziRicci system (3.3)-(3.5).

(i) Let $\left(h_{i j}^{a, \varepsilon}, \kappa_{l b}^{a, \varepsilon}\right)$ be a sequence of solutions to system (3.3)-(3.5), which is uniformly bounded in $L^{p}, p>2$. Then the weak limit vector field $\left(h_{i j}^{a}, \kappa_{l b}^{a}\right)$ of the sequence $\left(h_{i j}^{a, \varepsilon}, \kappa_{l b}^{a, \varepsilon}\right)$ in $L^{p}$ is still a solution to system (3.3)-(3.5).

(ii) There exists a minimizer $\left(h_{i j}^{a}, \kappa_{l b}^{a}\right)$ for the minimization problem:

$$
\min _{S}\|(h, \kappa)\|_{L^{p}(\Omega)}^{p}:=\min _{S} \int_{\Omega} \sqrt{|g|}\left(\left|h_{i j} h_{i j}\right|^{\frac{p}{2}}+\left|\kappa_{l b} \kappa_{l b}\right|^{\frac{p}{2}}\right) d x
$$

where $S$ is the set of weak solutions to system (3.3)-(3.5).

This weak continuity and rigidity theorem is a reminiscence of the polyconvexity theory in nonlinear elasticity by Ball [2, for which the rigidity of elastic bodies can be achieved.

The proof of this theorem is based on the following observations on 
the div-curl structure of the Gauss-Codazzi-Ricci system:

$$
\begin{aligned}
& \operatorname{div}(\overbrace{\underbrace{0, \cdots, 0, h_{l j}^{a, \varepsilon}}_{l}, 0, \cdots,-h_{k j}^{a, \varepsilon}}^{k}, 0, \cdots, 0)=R_{1}, \\
& \operatorname{curl}\left(h_{1 i}^{a, \varepsilon}, h_{2 i}^{a, \varepsilon}, \cdots, h_{d i}^{a, \varepsilon}\right)=R_{2}, \\
& \operatorname{div}(\overbrace{\underbrace{0, \cdots, 0, \kappa_{l b}^{a, \varepsilon}}_{l}, 0, \cdots,-\kappa_{k b}^{a, \varepsilon}}^{k}, 0, \cdots, 0)=R_{3}, \\
& \operatorname{curl}\left(\kappa_{1 b}^{a, \varepsilon}, \kappa_{2 b}^{a, \varepsilon}, \cdots, \kappa_{d b}^{a, \varepsilon}\right)=R_{4}, \\
& \operatorname{div}(\overbrace{\underbrace{0, \cdots, 0, h_{l i}^{b, \varepsilon}}_{l}, 0, \cdots,-h_{k i}^{b, \varepsilon}}^{k}, 0, \cdots, 0)=R_{5} \text {, } \\
& \operatorname{curl}\left(h_{1 i}^{b, \varepsilon}, h_{2 i}^{b, \varepsilon}, \cdots, h_{d i}^{b, \varepsilon}\right)=R_{6}, \\
& \operatorname{div}(\overbrace{l}^{\overbrace{0, \cdots, 0, \kappa_{l c}^{b, \varepsilon}}^{0, \cdots}, 0, \cdots,-\kappa_{k c}^{b, \varepsilon}}, 0, \cdots, 0)=R_{7}, \\
& \operatorname{curl}\left(\kappa_{1 c}^{b, \varepsilon}, \kappa_{2 c}^{b, \varepsilon}, \cdots, \kappa_{d c}^{b, \varepsilon}\right)=R_{8},
\end{aligned}
$$

and $R_{j}, j=1,2, \cdots, 8$, are confined in a compact set in $H_{l o c}^{-1}(\Omega)$.

Then employing the Murat-Tartar's div-curl lemma directly yields

$$
\begin{aligned}
& h_{l j}^{a, \varepsilon} h_{k i}^{a, \varepsilon}-h_{k j}^{a, \varepsilon} h_{l i}^{a, \varepsilon} \rightarrow h_{l j}^{a} h_{k i}^{a}-h_{k j}^{a} h_{l i}^{a}, \\
& h_{l j}^{a, \varepsilon} h_{k i}^{b, \varepsilon}-h_{k j}^{a, \varepsilon} h_{l i}^{b, \varepsilon} \rightarrow h_{l j}^{a} h_{k i}^{b}-h_{k j}^{a} h_{l i}^{b}, \\
& \kappa_{k b}^{a, \varepsilon} \kappa_{l c}^{b, \varepsilon}-\kappa_{l b}^{a, \varepsilon} \kappa_{k c}^{b, \varepsilon} \rightarrow \kappa_{k b}^{a} \kappa_{l c}^{b}-\kappa_{l b}^{a} \kappa_{k c}^{b}, \\
& \kappa_{k b}^{a, \varepsilon} h_{l i}^{b, \varepsilon}-\kappa_{l b}^{a, \varepsilon} h_{k i}^{b, \varepsilon} \rightarrow \kappa_{k b}^{a} h_{l i}^{b}-\kappa_{l b}^{a} h_{k i}^{b}
\end{aligned}
$$

in the sense of distributions as $\varepsilon \rightarrow 0$, which implies the weak continuity and rigidity of system (3.3)-(3.5) and corresponding isometric embeddings.

A compactness framework for the Gauss-Codazzi-Ricci system (3.3)(3.5) has also established in [23: Given any sequence of approximate solutions to this system which is uniformly bounded in $L^{2}$ and has reasonable bounds on the errors made in the approximation (the errors are confined in a compact subset of $H_{\text {loc }}^{-1}$ ), then the approximating sequence has a weakly convergent subsequence whose limit is still a solution of system (3.3)-(3.5) .

These results indicate that the weak limit of isometrically embedded surfaces is still an isometrically embedded surface in $\mathbb{R}^{d}$ for any Rie- 
mann curvature tensor $\left\{R_{i j k l}\right\}$ without restriction, which is the rigidity property of embedded surfaces in geometry.

Acknowledgements. The materials presented above include direct and/or indirect contributions of my collaborators Xiaxi Ding, Feimin Huang, Philippe LeFloch, Bang-He Li, Tianhong Li, Yunguang Lu, Peizhu Luo, Mikhail Perepelitsa, Marshall Slemrod, Dehua Wang, Tian-Yi Wang, Yongqian Zhang, Dianwen Zhu, among others.

\section{References}

[1] G. Alberti and S. Müller, A new approach to variational problems with multiple scales. Comm. Pure Appl. Math. 54 (2001), 761-825.

[2] J. M. Ball, Convexity conditions and existence theorems in nonlinear elasticity. Arch. Rational Mech. Anal. 63 (1976/77), 337-403.

[3] J. M. Ball, A version of the fundamental theorem for Young measures. Lecture Notes in Phys. 344, pp. 207-215, Springer: Berlin, 1989.

[4] S. Bianchini and A. Bressan, Vanishing viscosity solutions of nonlinear hyperbolic systems. Ann. of Math. (2), 161 (2005), 223-342.

[5] A. Bressan, Hyperbolic Systems of Conservation Laws: The OneDimensional Cauchy Problem. Oxford University Press: Oxford, 2000.

[6] M. Briane, J. Casado-Daz, and F. Murat, The div-curl lemma "trente ans aprs": an extension and an application to the Gconvergence of unbounded monotone operators. J. Math. Pures Appl. (9) 91 (2009), 476-494.

[7] R. L. Bryant, P. A. Griffiths, and D. Yang, Characteristics and existence of isometric embeddings. Duke Math. J. 50 (1983), 893994.

[8] G.-Q. Chen, Convergence of the Lax-Friedrichs scheme for isentropic gas dynamics (III). Acta Math. Sci. 6B (1986), 75-120 (in English); 8A (1988), 243-276 (in Chinese).

[9] G.-Q. Chen, Remarks on spherically symmetric solutions of the compressible Euler equations. Proc. Royal Soc. Edinburgh, 127A (1997), 243-259.

[10] G.-Q. Chen, Euler Equations and Related Hyperbolic Conservation Laws. In: Handbook of Differential Equations: Evolutionary Differential Equations, Vol. 2, pp. 1-104, 2005, Eds. C. M. Dafermos and E. Feireisl, Elsevier Science B.V: Amsterdam, The Netherlands. 
[11] G.-Q. Chen and C. Christoforou, Solutions for a nonlocal conservation law with fading memory. Proc. Amer. Math. Soc. 135 (2007), no. $12,3905-3915$.

[12] G.-Q. Chen, C. M. Dafermos, M. Slemrod, M., and D. Wang, On two-dimensional sonic-subsonic flow. Commun. Math. Phys. 271 (2007), 635-647.

[13] G.-Q. Chen, F.-M. Huang, and T.-Y. Wang, Subsonic-sonic limit of approximate solutions to multidimensional steady Euler equations. Arch, Rational Mech. Anal. 2015 (to appear); arXiv:1311.3985.

[14] G.-Q. Chen and P. G. LeFloch, Compressible Euler equations with general pressure law, Arch. Rational Mech. Anal. 153 (2000), 221259; Existence theory for the isentropic Euler equations, Arch. Rational Mech. Anal. 166 (2003), 81-98.

[15] G.-Q. Chen and T.-H. Li, Well-posedness for two-diemnsonal steady supersonic Euler flows past a Lipschitz wedge. J. Diff. Eqs. 244 (2008), 1521-1550.

[16] G.-Q. Chen, B.-H. Li, and T.-H. Li, Entropy solutions in $L^{\infty}$ for the Euler equations in nonlinear elastodynamics and related equations. Arch. Rational Mech. Anal. 170 (2003), 331-357.

[17] G.-Q. Chen and Y.-G. Lu, The study on application way of the compensated compactness theory. Chinese Sci. Bull. 33 (1988), 641644 (in Chinese); 34 (1989), 15-19 (in English).

[18] G.-Q. Chen and M. Perepelitsa, Vanishing viscosity limit of the Navier-Stokes equations to the Euler equations for compressible fluid flow. Comm. Pure Appl. Math. 63 (2010), 1469-1504.

[19] G.-Q. Chen and M. Perepelitsa, Shallow water equations: viscous solutions and inviscid limit. Z. Angew. Math. Phys. 63 (2012), 10671084 .

[20] G.-Q. Chen and M. Perepelitsa, Vanishing viscosity solutions of the compressible Euler equations with spherically symmetry and large initial data. Comm. Math. Phys. 338 (2015), 771-800.

[21] G.-Q. Chen, M. Slemrod, and D. Wang, Vanishing viscosity method for transonic flow. Arch. Rational Mech. Anal. 189 (2008), 159-188.

[22] G.-Q. Chen, M. Slemrod, and D. Wang, Isometric immersions and compensated compactness. Comm. Math. Phys. 294 (2010), 411437.

[23] G.-Q. Chen, M. Slemrod, and D. Wang, Weak continuity of the Gauss-Codazzi-Ricci system for isometric embedding. Proc. Amer. Math. Soc. 138 (2010), 1843-1852. 
[24] G.-Q. Chen, Y.-Q. Zhang, and D.-W. Zhu, Existence and stability of supersonic Euler flows past Lipschitz wedges. Arch. Rational Mech. Anal. 181 (2006), 261-310.

[25] R. Courant and K. O. Friedrichs, Supersonic Flow and Shock Waves. Springer-Verlag: New York, 1948.

[26] C. M. Dafermos, Solutions in $L^{\infty}$ for a conservation law with memory. Analyse Mathématique et Applications, 117-128, GauthierVillars, Montrouge, 1988.

[27] C. M. Dafermos, Hyperbolic Conservation Laws in Continuum Physics. Springer-Verlag: Berlin, 2010.

[28] C. De Lellis and L. Székelyhidi, Jr., On admissibility criteria for weak solutions of the Euler equations. Arch. Rational Mech. Anal. 195 (2010), 225-260.

[29] C. De Lellis and L. Székelyhidi, Jr., The h-principle and the equations of fluid dynamics. Bull. Amer. Math. Soc. (N.S.) 49 (2012), 347-375.

[30] X. Ding, G.-Q. Chen, and P. Luo, Convergence of the Lax-Friedrichs scheme for the isentropic gas dynamics (I)-(II), Acta Math. Sci. 5B (1985), 483-500, 501-540 (in English); 7A (1987), 467-480; 8A (1989), 61-94 (in Chinese); Convergence of the fractional step LaxFriedrichs scheme and Godunov scheme for the isentropic system of gas dynamics, Comm. Math. Phys. 121 (1989), 63-84.

[31] R. J. DiPerna, Decay of solutions of hyperbolic systems of conservation laws with a convex extension. Arch. Rational Mech. Anal. 64 (1977), 1-46.

[32] R. J. DiPerna, Convergence of the viscosity method for isentropic gas dynamics. Commun. Math. Phys. 91 (1983), 1-30.

[33] R. J. DiPerna, Convergence of approximate solutions to conservation laws. Arch. Rational Mech. Anal. 82 (1983), 27-70.

[34] R. J. DiPerna, Measure-valued solutions to conservation laws. Arch. Rational Mech. Anal. 88 (1985), 223-270.

[35] R. J. DiPerna, Compensated compactness and general systems of conservation laws. Trans. Amer. Math. Soc. 292 (1985), 383-420.

[36] N. V. Efimov, The impossibility in Euclideam 3-space of a complete regular surface with a negative upper bound of the Gaussian curvature. Dokl. Akad. Nauk SSSR (N.S.), 150 (1963), 1206-1209; Soviet Math. Dokl. 4 (1963), 843-846.

[37] L. C. Evans, Weak Convergence Methods for Nonlinear Partial Differential Equations. CBMS-RCSM, 74, AMS: Providence, RI, 1990. 
[38] D. Gilbarg, The existence and limit behavior of the one-dimensional shock layer. Amer. J. Math. 73 (1951), 256-274.

[39] J. Glimm, Solutions in the large for nonlinear hyperbolic systems of equations. Comm. Pure Appl. Math. 18 (1965), 697-715.

[40] J. Glimm and P. D. Lax, Decay of solutions of systems of hyperbolic conservation laws. Bull. Amer. Math. Soc. 73 (1967), no. 105.

[41] M. Gromov, Partial Differential Relations. Springer-Verlag: Berlin, 1986.

[42] G. Guderley, Starke kugelige und zylindrische Verdichtungsstosse inder Nahe des Kugelmittelpunktes bzw. der Zylinderachse. Luftfahrtforschung 19 (1942), no. 9, 302-311.

[43] C.M.I.O. Guès, G. Métivier, M. Williams, and K. Zumbrun, NavierStokes regularization of multidimensional Euler shocks. Ann. Sci. École Norm. Sup. (4) 39 (2006), 75-175.

[44] M. Günther, Zum Einbettungssatz von J. Nash. (German) [On the embedding theorem of J. Nash]. Math. Nachr. 144 (1989), 165-187.

[45] Q. Han and J.-X. Hong, Isometric Embedding of Riemannian Manifolds in Euclidean Spaces. AMS: Providence, RI, 2006.

[46] D. Hoff, Global solutions of the equations of one-dimensional, compressible flow with large data and forces, and with differing end states. Z. Angew. Math. Phys. 49 (1998), 774-785.

[47] D. Hoff and T.-P. Liu, The inviscid limit for the Navier-Stokes equations of compressible, isentropic flow with shock data. Indiana Univ. Math. J. 38 (1989), 861-915.

[48] H. Holden and N. H. Risebro, Front Tracking for Hyperbolic Conservation Laws. Springer: New York, 2011.

[49] E. Hopf, The partial differential equation $u_{t}+u u_{x}=u_{x x}$. Comm. Pure Appl. Math. 3 (1950), 201-230.

[50] F. Huang and Z. Wang, Convergence of viscosity solutions for isothermal gas dynamics. SIAM J. Math. Anal. 34 (2002), 595-610.

[51] M. Janet, Sur la possibilité de plonger un espace riemannian donné dans un espace euclidien. Ann. Soc. Pol. Math. 5 (1926), 38-43.

[52] I. Kanel, On a model system of equations for one-dimensional gas motion. Differ. Uravn. 4 (1968), 721-734.

[53] S. N. Kruzkov, First order quasilinear equations with several independent variables (Russian). Mat. Sb. (N.S.) 81 (123) (1970), 228255 . 
[54] P. D. Lax, Shock wave and entropy. In: Contributions to Functional Analysis, ed. E.A. Zarantonello, 603-634, Academic Press: New York, 1971.

[55] P. D. Lax, Hyperbolic Systems of Conservation Laws and the Mathematical Theory of Shock Waves. CBMS Regional Conference Series in Mathematics, No. 11. Philadelphia: SIAM, 1973.

[56] P. G. LeFloch, Hyperbolic Systems of Conservation Laws: The Theory of Classical and Nonclassical Shock Waves. Birkhäuser Verlag: Basel, 2002.

[57] P. G. LeFloch and V. Shelukhin, Symmetries and local solvability of the isothermal gas dynamics equations. Arch. Rational Mech. Anal. 175 (2005), 389-430.

[58] P. G. LeFloch and M. Westdickenberg, Finite energy solutions to the isentropic Euler equations with geometric effects. J. Math. Pures Appl. 88 (2007), 386-429.

[59] T.-P. Liu, Admissible solutions of hyperbolic conservation laws. Mem. Amer. Math. Soc. 30 (1981), no. 240.

[60] T.-P. Liu and T. Yang, Well-posedness theory for hyperbolic conservation laws. Comm. Pure Appl. Math. 52 (1999), 1553-1586.

[61] P.-L. Lions, B. Perthame, and P. E. Souganidis, Existence and stability of entropy solutions for the hyperbolic systems of isentropic gas dynamics in Eulerian and Lagrangian coordinates. Comm. Pure Appl. Math. 49 (1996), 599-638.

[62] P.-L. Lions, B. Perthame, and E. Tadmor, Kinetic formulation of the isentorpic gas dynamics and p-systems. Comm. Math. Phys. 163 (1994), 415-431.

[63] S. Mardare, The fundamental theorem of surface theory for surfaces with little regularity. J. Elasticity, 73 (2003), 251-290.

[64] S. Mardare, On Pfaff systems with $L^{p}$ coefficients and their applications in differential geometry. J. Math. Pure Appl. 84 (2005), 1659-1692.

[65] C. S. Morawetz, On a weak solution for a transonic flow problem. Comm. Pure Appl. Math. 38 (1985), 797-818.

[66] C. S. Morawetz, An alternative proof of DiPerna's theorem. Comm. Pure Appl. Math. 44 (1991), 1081-1090.

[67] C. S. Morawetz, On steady transonic flow by compensated compactness. Methods Appl. Anal. 2 (1995), 257-268.

[68] F. Murat, Compacité par compensation. Ann. Scuola Norm. Sup. Pisa Sci. Fis. Mat. 5 (1978), 489-507. 
[69] F. Murat, Compacité par compensation. II (French). In: Proceedings of the International Meeting on Recent Methods in Nonlinear Analysis (Rome, 1978), pp. 245-256, Pitagora, Bologna, 1979.

[70] F. Murat, L'injection du cône positif de $H^{-1}$ dans $W^{-1, q}$ est compacte pour tout $q<2$ (French). J. Math. Pures Appl. (9) 60 (1981), 309-322.

[71] F. Murat, Compacité par compensation: condition nécessaire et suffisante de continuité faible sous une hypothse de rang constant (French). Ann. Scuola Norm. Sup. Pisa Cl. Sci. (4) 8 (1981), no. 1, 69-102.

[72] F. Murat, A survey on compensated compactness. In: Contributions to Modern Calculus of Variations (Bologna, 1985), 145-183, Pitman Res. Notes Math. Ser. 148, Longman Sci. Tech., Harlow, 1987.

[73] J. Nash, The imbedding problem for Riemannian manifolds. Ann. Math. (2), 63 (1956), 20-63.

[74] O. A. Oleinik, Discontinuous solutions of non-linear differential equations. Usp. Mat. Nauk 12 (1957), 3-73. English translation: AMS Translations, Ser. II, 26, 95-172.

[75] B. Perthame and A. E. Tzavaras, Kinetic formulation for systems of two conservation laws and elastodynamics. Arch. Rational Mech. Anal. 155 (2000), 1-48.

[76] J. Rauch, BV estimates fail for most quasilinear hyperbolic systems in dimension greater than one. Comm. Math. Phys. 106 (1986), 481-484.

[77] S. Rosseland, The Pulsation Theory of Variable Stars. Dover Publications, Inc.: New York, 1964.

[78] M. E. Schonbek, Convergence of solutions to nonlinear dispersive equations. Comm. Partial Diff. Eqs. 7 (1982), 959-1000.

[79] D. Serre, La compacité par compensation pour les systèmes non linéaires de deux equations a une dimension d'espace. J. Math. Pures Appl. 65 (1987), 423-468.

[80] D. Serre and J. W. Shearer, Convergence with physical viscosity for nonlinear elasticity. Preprint, 1994 (unpublished).

[81] J. Smoller, Shock Waves and Reaction-Diffusion Equations. Second edition. Springer-Verlag: New York, 1994.

[82] E. Tadmor, M. Rascle, and P. Bagnerini, Compensated compactness for 2D conservation laws. J. Hyper. Diff. Eqs. 2 (2005), 697-712.

[83] L. Tartar, Compensated compactness and applications to partial differential equations. In: Research Notes in Mathematics, Non- 
linear Analysis and Mechanics, Herriot-Watt Symposium, Vol. 4, Knops R.J. ed., Pitman Press, 1979.

[84] L. Tartar, The compensated compactness method applied to systems of conservation laws. In: Systems of Nonlinear Partial Differential Equations (Oxford, 1982), 263-285, NATO Adv. Sci. Inst. Ser. C Math. Phys. Sci. 111, Reidel, Dordrecht, 1983.

[85] L. Tartar, Compacité par compensation: résultats et perspectives (French). In: Nonlinear Partial Differential Equations and Their Applications, Collège de France Seminar, Vol. IV (Paris, 1981/1982), 350-369, Res. Notes in Math. 84, Pitman, Boston, MA, 1983.

[86] L. Tartar, Oscillations in nonlinear partial differential equations: compensated compactness and homogenization. In: Nonlinear Systems of Partial Differential Equations in Applied Mathematics, Part 1 (Santa Fe, N.M., 1984), 243-266, Lectures in Appl. Math., 23, Amer. Math. Soc.: Providence, RI, 1986.

[87] L. Tartar, Discontinuities and oscillations. In: Directions in Partial Differential Equations (Madison, WI, 1985), 211-233, Publ. Math. Res. Center Univ. Wisconsin, 54, Academic Press, Boston, MA, 1987.

[88] L. Tartar, From Hyperbolic Systems to Kinetic Theory: A Personalized Quest. Lecture Notes of the Unione Matematica Italiana, 6. Springer-Verlag, Berlin; UMI, Bologna, 2008.

[89] L. Tartar, The General Theory of Homogenization: A Personalized Introduction. Lecture Notes of the Unione Matematica Italiana, 7. Springer-Verlag, Berlin; UMI, Bologna, 2009.

[90] A. I. Vol'pert, Spaces BV and quasilinear equations (Russian). Mat. Sb. (N.S.) 73 (115) (1967), 255-302.

[91] G. B. Whitham, Linear and Nonlinear Waves. John Wiley \& Sons: New York, 1974.

[92] S.-T. Yau, Review of geometry and analysis. In: Mathematics: Frontiers and Perspectives, pp. 353-401, International Mathematics Union, Eds. V. Arnold, M. Atiyah, P. Lax, and B. Mazur, AMS: Providence, 2000. 